\title{
A Fading-Resistant Method for RFID-Antenna Structural Mode Measurement
}

\author{
Antonis G. Dimitriou \\ Department of Electrical \& Computer Engineering, \\ Aristotle University of Thessaloniki \\ Thessaloniki, Greece \\ antodimi@auth.gr
}

\author{
* John Kimionis, " Aggelos Bletsas \\ Department of Electronic \& Computer Engineering, \\ Technical University of Crete \\ Chania, Greece \\ *ikimionis@isc.tuc.gr, ${ }^{\#}$ aggelos@telecom.tuc.gr
}

\author{
John N. Sahalos \\ Department of Electrical \& Computer Engineering, \\ University of Nicosia \\ Nicosia, Cyprus \\ sahalos.j@unic.ac.cy
}

\begin{abstract}
In this paper, a prototype device to measure the tagantenna's structural mode term is proposed. The device comprises a switching transistor, controlled by a microcontroller, and a reverse-biased varactor diode connected to the transistor. The antenna's load is selectively modified by applying the appropriate DC voltage on the varactor. Antenna's structural mode is evaluated by measuring the backscattered modulated field for three states of the varactor. In contrast to prior art: $i$ ) the proposed device can be conveniently used to measure the structural mode of any antenna, via an $R F$ connector, without the need to successively attach different loads to the antenna under test, ii) measurements can be performed under fading/changing conditions, as fading-effects are removed, as demonstrated herein. The device can be used to evaluate backscatter communications for different tag-loading. Furthermore, it could operate as an RF sensor, where the sensor's voltage "drives" the varactor's capacitance.
\end{abstract}

\section{RFID; antenna; structural-mode; backscattering}

\section{INTRODUCTION}

Performance of an RFID system greatly depends on proper load selection for the two modulation states [1]-[3]. Such selection should ensure enough backscattered power at the reader and sufficient separation in the complex plane of the backscattered fields that correspond to the two states of tag loading. Achievement of optimal tag-loading depends greatly on the structural-mode term of the antenna [3]. This term properly corrects the backscattered field from a tag-antenna that is modeled by a Thévenin's equivalent circuit [4]-[6]. It has been shown in [3] that proper tag-loading, exploiting the structural term, could lead to a $7 \mathrm{~dB}$ gain of the mean backscattered power, thus aiding the reader to cope with very weak modulated signals and allowing it to operate within its spurious free dynamic range.

The typical method for measuring tag-antenna's structural mode is by successively connecting the antenna to three different known loads (typically "open", "short" and "conjugate match" are selected) and measuring the antennaload RCS in an anechoic chamber for each case [4], [7]. This method is impractical, because the antenna must be consecutively soldered and unsoldered to three different loads and the measurements require identical anechoic chamber conditions, which are not widely available.

In this work, we propose the design and construction of a single prototype circuit that measures any antenna's structural mode under any measurement-conditions. Hence, the same circuit can be attached to any antenna and measurements can be conducted even in a typical indoor "rich-scattering" environment.

The key-element of the proposed design is a varactor diode that is used to selectively change the load in the "on" state of a switching transistor. The transistor is controlled by a microcontroller and the reverse-voltage on the diode by a configurable DC-source. All parameters of the circuit are measured, using a vector network analyzer. As a consequence the circuit will produce configurable loads, which allow measurement of the antenna's structural mode term, as explained in this work. Furthermore, a technique to eliminate the effects of fading is proposed that exploits the "low" state of the transistor, which is common in all measurements. Since the proposed circuit "produces" a continuous variation of a modified reflection coefficient, it can also be used for experimentation with different combinations of modulation states. Finally, assuming that the DC voltage could be driven by a sensor, the proposed circuit can be alternatively used as an RFID UHF sensor.

\section{ANTENNA BACKSCATTERING}

The current at the Thévenin's equivalent circuit of an antenna connected to a load does not quantify its backscattered field. To properly evaluate the backscattered field, we should 
calculate a "modified" tag's radar cross section $\sigma$, derived in [5]:

$$
\begin{gathered}
\sigma=\frac{\lambda^{2}}{4 \pi} G_{t a g}^{2}\left|\Gamma-A_{s}\right|^{2}, \\
\Gamma=\frac{Z_{L}-Z_{a}^{*}}{Z_{L}+Z_{a}}
\end{gathered}
$$

where $\lambda$ is the wavelength at the operating frequency, $G_{t a g}$ is the tag's antenna gain and $A_{s}$ is a complex parameter independent of the load that quantifies the structural mode term of the antenna. $\Gamma$ expresses a modified reflection coefficient, defined in (2), where $Z_{L}, Z_{a}$ are the load's and the antenna's impedance respectively.

The term $A_{s}$ can be measured or simulated for any tag's antenna by evaluating or measuring the radar cross section for three different loads as demonstrated in [7]. Measurement of $A_{s}$ for tag-antennas can be found in [4], [7]. In this work, we will demonstrate a more practical method to measure the tagantenna's structural term $A_{s}$.

\section{MEASUREMENT OF THE StRUCTURAL MODE}

\section{A. Simplified Circuit}

The proposed circuit is demonstrated in Figure 1. It consists of three blocks, shown in dash-dotted rectangles: the antenna, the transistor (its simplified equivalent at the "on" state is demonstrated in Figure 1) and the tunable load. The total load $Z_{L}$, connected to the antenna at the "on" state of the transistor, equals the sum of the impedance of the transistor plus the impedance of the DC controlled load.

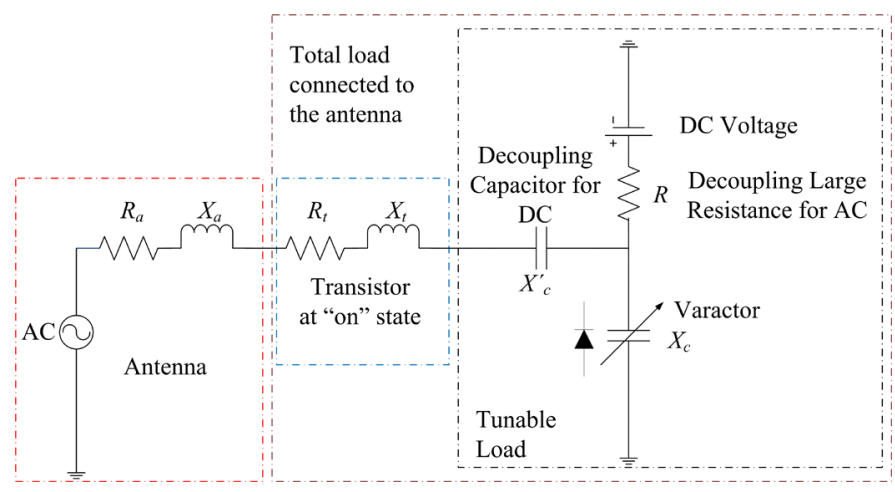

Figure 1. Block diagramm of the proposed circuit.

The AC source represents the field that is captured by the tag-antenna. The complex impedance of the tag-antenna is represented by a resistor connected in series to an inductor, which is typical for passive RFID tag antennas [8]- [10]. In such cases, power matching to the rectifier (capacitive) at the input of the chip is sought. The proposed method can be directly applied for capacitive antennas as well.
The antenna in Figure 1 is connected to a transistor, controlled by a microcontroller. The PCB with the transistor and the microcontroller are shown in Figure 2. The test-bed for measurements is shown in Figure 3. Backscattered measurements from the "switching" transistor of Figure 2, as captured by the URSP SDR of Figure 3, are demonstrated in Figure 4. The constructed device, shown in Figure 2, does not include the DC-controlled "load" shown in Figure 1 that will allow measurement of the antenna's structural mode, as will be shown next.

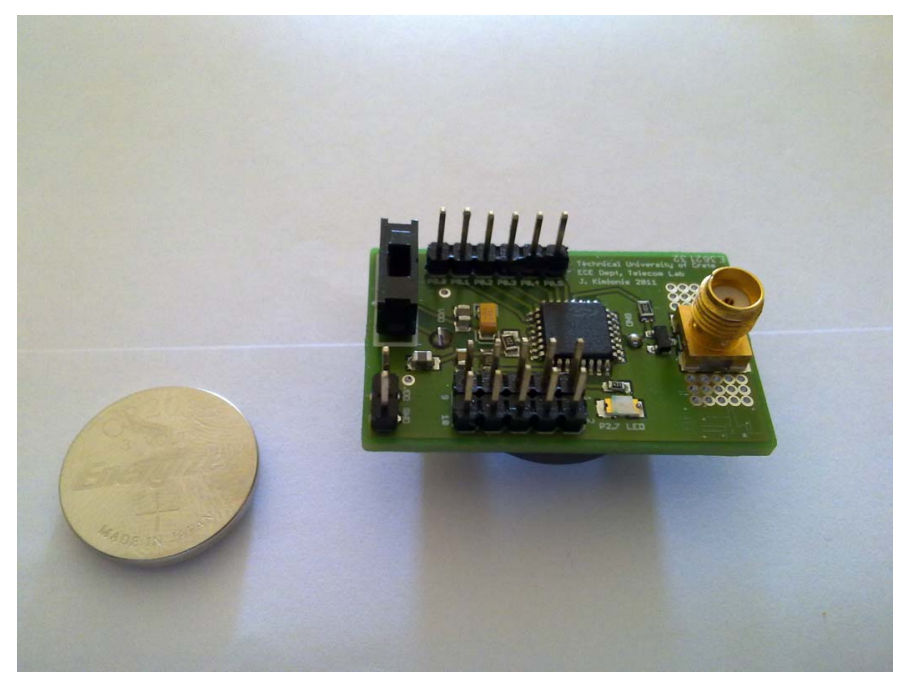

Figure 2. PCB including the microcontroller with the transistor (semipassive tag).

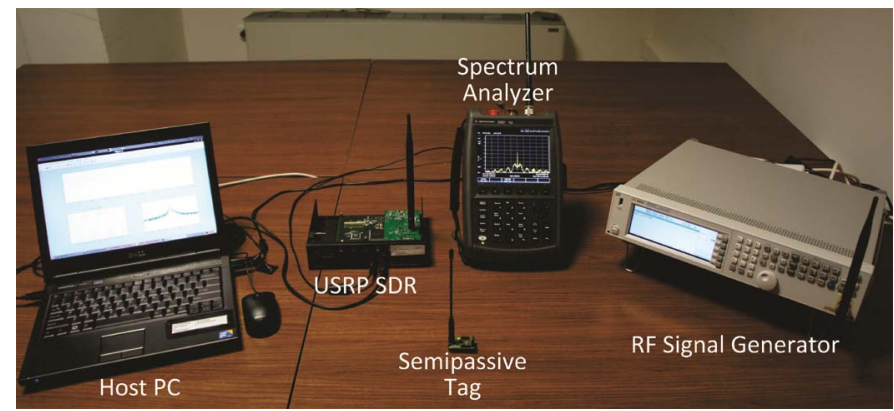

Figure 3. Photo of the measurements' setup.

We propose attaching a varactor at the "on" state of the transistor with variable impedance represented by $X_{C}$. The capacitance of commercial SMD UHF varactors ranges from $0.68 \mathrm{pF}$ to $40 \mathrm{pF}$, as the inverse $\mathrm{DC}$ voltage ranges from $28 \mathrm{~V}$ to $1 \mathrm{~V}$ respectively [11]-[13]. Hence, the impedance $X_{C}$ at $930 \mathrm{MHz}$ changes from $250 \mathrm{Ohms}$ down to $4 \mathrm{Ohms}$ respectively. Capacitor $X_{C}{ }^{\prime}$ is used to decouple the DC voltage applied at the diode from the transistor. Resistor $R$ is employed to decouple the $\mathrm{AC}$ current of the circuit from the DC source.

\section{B. Basic operation of the circuit}

As we control the inverse DC voltage at the diode, we change the load at the "on" state of the transistor. Hence, we change the modified reflection coefficient, expressed in (2). Thus, we alter the backscattered RCS $\sigma^{o n}$ at the "on" state of the transistor, given in (1). Since the complex impedance of all 
elements of the circuit of Figure 1 can be measured, as will be shown in the following sections, we can calculate the term $A_{s}$ in (1) by applying three different DC voltages at the varactor. Analytical solutions are provided in the following sections.

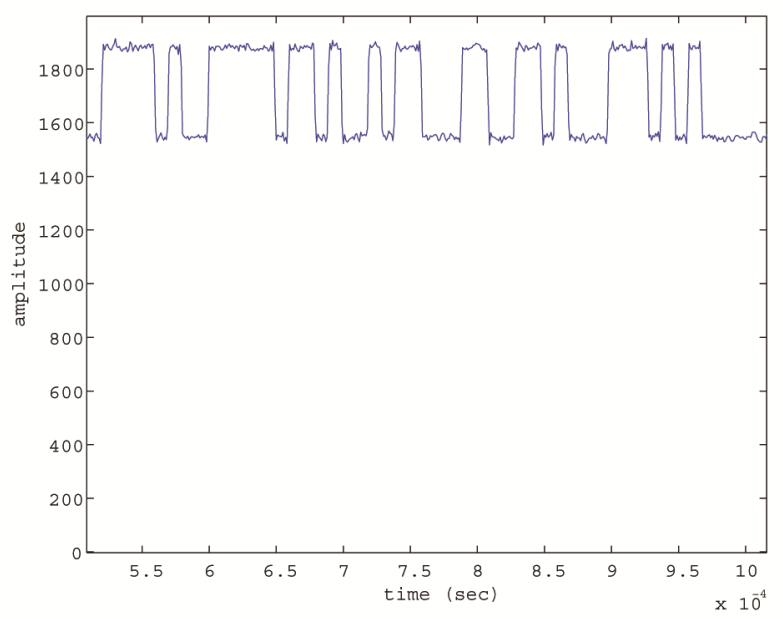

Figure 4. Measured backscattered field by the switching transistor.

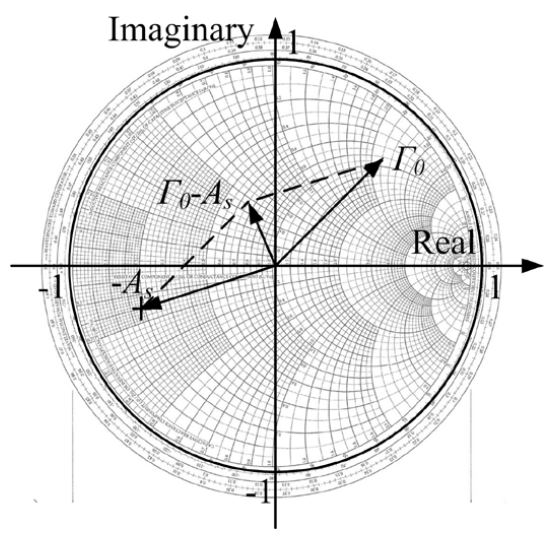

Figure 5. Modified reflection coefficient and structural term at state " 0 " of the transistor.

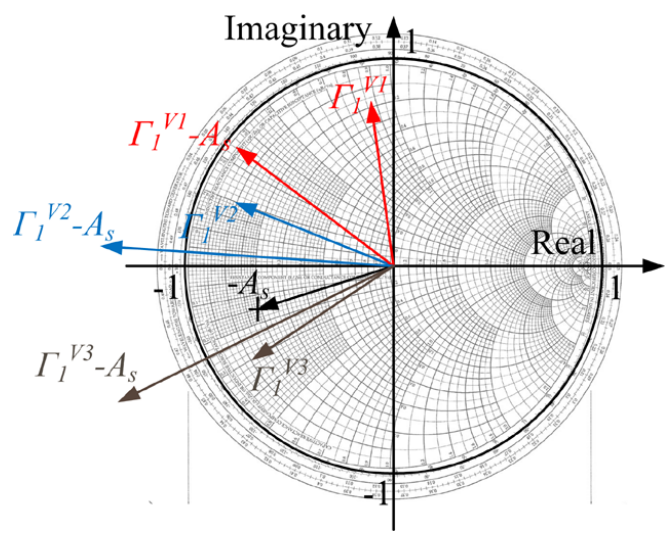

Figure 6. Modified reflection coefficients and structural term at state "1" of the transistor for three different voltage-controlled loads.

\section{Calculation of the structural term of the antenna}

The above process is visually presented in Figures 5-7. In Figure 5 , the unknown vector " $-A_{s}$ " is shown in the complex plane. The modified reflection coefficient $\Gamma_{0}$ is measured with a vector network analyzer for the "off" state of the transistor and depicted on a Smith chart. The magnitude of the vectorsum $\left|\Gamma_{0}-A_{s}\right|$ sizes the backscattered power at state "off" of the transistor, according to (1). In Figure 6, the transistor is at the "on" state and by applying three different DC-voltages, we produce three different reflection-coefficients denoted as $\Gamma_{l}{ }^{V i}$, $i=1,2,3$. The magnitude of the resulting vectors $\left|\Gamma_{I}^{V i}-A_{s}\right|$ sizes the backscattered power at the "on" state of the transistor.

Consider that the microcontroller of Figure 2 drives the transistor to backscatter the same bitstream 3 times. Each of the 3 times the DC-voltage that controls the load takes the values $V_{1}, V_{2}$ and $V_{3}$ respectively that produce the complex reflection coefficients $\Gamma_{l}^{V i}, i=1,2,3$ that were shown in Figure 5 . Assuming identical measurement-propagation-channelconditions in the three cases, the measured backscattered received voltage would look like the three bitstreams of Figure 7 (the corresponding actual measurements from a transistor were shown in Figure 4). In these bitstreams, "State 0" of the transistor is common (since different tag loading does not affect this state), while "State 1" changes proportionally to $\mid \Gamma_{l}^{V i}$ $-A_{s} \mid$.

Transmission of the same bitstream for 3 different loads

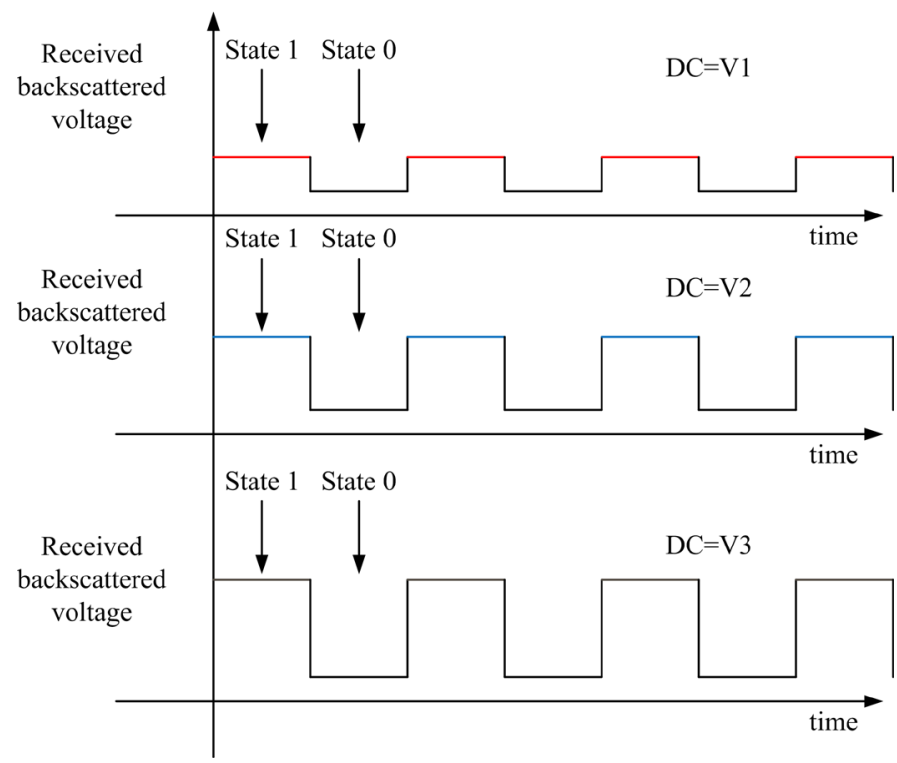

Figure 7. Measured bitstream for 3 voltages applied to the varactor.

Let $V_{0}^{j}$ denote the measured backscattered voltage of "State 0 " for the $j^{\text {th }}$ transmission of the bitstream $(j=1,2,3)$ and $V_{1}^{j}$ denote the corresponding voltage for "State 1" (Figure 7). The measured voltage for the two states is then:

$$
V_{0}^{j}=a_{j} \sqrt{\sigma_{0}},
$$




$$
V_{1}^{j}=a_{j} \sqrt{\sigma_{1}^{j}}
$$

The term $a_{j}$ sizes the common terms in each transmission, including transmission power, antenna gains, distance between transmitter and receiver and fading. In (3) and (4), we have defined $a_{j}$ to be constant for each measurement, but different among successive measurements. For a given measurement, transmission power, antenna gains and distance are common for both states of the transistor. Furthermore, two successive bits suffer the same fading. However, for two different measurements, conducted in different times, even if the setup is the same (power, distance) the channel may have changed (due to temporal fading). Hence, $a_{j}(j=1,2,3)$ is expected to be different. We will show next how we will take advantage of the common reflection coefficient at "State 0" in the 3 measurements, in order to eliminate the different fading conditions during the 3 measurements. Let:

$$
\Gamma_{1}^{V i}=x_{i}+j y_{i}
$$

$$
A_{s}=x+j y
$$

To avoid the effects of fading, we define:

$$
V^{j}=\frac{V_{1}^{j}}{V_{0}^{j}}=\frac{\sqrt{\sigma_{1}^{j}}}{\sqrt{\sigma_{0}}}
$$

So for each measured bitstream, we divide the measured voltage at state 1 to the measured voltage at state 0 , where we know that the reflection coefficient $\Gamma_{0}$ is common, resulting to the common RCS $\sigma_{0}$, defined in (1). The measured quantity $V^{j}$ no longer includes the fading parameter $a_{j}$ and the only reasonable assumption, so far, is that successive bits within the same bitstream have suffered the same fading. Then we create our system of equations by carrying out the following divisions and substituting $\sigma_{1}^{j}$ from (1), (5) and (6):

$$
\begin{aligned}
& k_{12}=\left(\frac{V^{1}}{V^{2}}\right)^{2}=\frac{\sigma_{1}^{1}}{\sigma_{1}^{2}}=\frac{\left(x-x_{1}\right)^{2}+\left(y-y_{1}\right)^{2}}{\left(x-x_{2}\right)^{2}+\left(y-y_{2}\right)^{2}} \\
& k_{13}=\left(\frac{V^{1}}{V^{3}}\right)^{2}=\frac{\sigma_{1}^{1}}{\sigma_{1}^{3}}=\frac{\left(x-x_{1}\right)^{2}+\left(y-y_{1}\right)^{2}}{\left(x-x_{3}\right)^{2}+\left(y-y_{3}\right)^{2}} \\
& k_{23}=\left(\frac{V^{2}}{V^{3}}\right)^{2}=\frac{\sigma_{1}^{2}}{\sigma_{1}^{3}}=\frac{\left(x-x_{2}\right)^{2}+\left(y-y_{2}\right)^{2}}{\left(x-x_{3}\right)^{2}+\left(y-y_{3}\right)^{2}}
\end{aligned}
$$

Exact solution of the system of equations (8)-(10) for the unknown $x, y$ has been presented in our prior work: see appendix-equations A4-A10 in [1]. In the analysis so far we have assumed that the loads at the "State 1" of the transistor are known. Indeed, by implementing a vector network analyzer to characterize the transistor and the antenna, all complex loads have been measured in the frequency domain, as presented in the following section.

\section{CIRCUIT'S PARAMETERS}

In the proposed circuit, we change the capacitance of the varactor, in order to change the modified reflection coefficient in (2) and thus change the amplitude of the modulated bistream at "State 1" of the switching transistor. In order to accomplish significant "changes" in the amplitude of the backscattered stream, we should carefully select the values of the circuit's components. In this section, we focus on proper selection of the parameters of the circuit.

The series combination of the decoupling capacitor $C^{\prime}$ with the varactor capacitance $C$ produce an equivalent capacitance $C_{e q}$ given by:

$$
C_{e q}=\frac{C^{\prime} C}{C^{\prime}+C}
$$

$C^{\prime}$ should be much larger than $C\left(C^{\prime}>>C\right)$, in order to maintain the range of variation of the load (produced by the changing $C$ ) as much as possible. We denote by $X$ the equivalent impedance of the series combination of $C^{\prime}, C$. The magnitude $\mid \Pi$ and phase $\varphi_{\Gamma}$ of the modified reflection coefficient, given in (2), for the parameters of the circuit shown in Figure 1 is given by:

$$
\begin{gathered}
|\Gamma|=\left[\frac{\left(R_{t}-R_{a}\right)^{2}+\left(X-X_{t}-X_{a}\right)^{2}}{\left(R_{t}+R_{a}\right)^{2}+\left(X-X_{t}-X_{a}\right)^{2}}\right] \\
\varphi_{\Gamma}=\arctan \left[\frac{-2 R_{a}\left(X-X_{t}-X_{a}\right)}{\left(X-X_{t}-X_{a}\right)^{2}-\left(R_{a}^{2}-R_{t}^{2}\right)}\right]
\end{gathered}
$$

In order to rotate the vector of the reflection coefficient $\Gamma$ in $(0,2 \pi)$, the nominator (imaginary axis) and the denominator (real axis) of (13) should take all combinations of $\{ \pm, \pm\}$ signs. It is found that the denominator becomes negative if and only if:

$$
R_{a}>R_{t}
$$

We substitute $R^{\prime}=\left(R_{a}^{2}-R_{t}^{2}\right)^{1 / 2}$ and summarize the signchanges of the real and imaginary part of $\Gamma$, as $X$ changes from its minimum to its maximum value, in Table I. For example, from Table I, we find that if we want $\Gamma$ to take values in the $2^{\text {nd }}$ quadrant, we should tune (by the applied DC voltage) $X$ in the following range: $X_{t}+X_{a}-R^{\prime}<X<X_{t}+X_{a}$. 
TABLE I. Phase of $\Gamma$ By ChangING LOAD $X$

\begin{tabular}{|c|c|c|c|c|}
\hline$X$ & \multicolumn{3}{|c|}{$X_{t}+X_{a}-R^{\prime}$} & $X_{t}+X_{a}$ \\
\hline Real & + & - & - & + \\
\hline Imag & + & + & - & - \\
\hline Quadrant & $\mathbf{1}^{\text {st }}$ & $2^{\text {nd }}$ & $3^{\text {rd }}$ & $4^{\text {th }}$ \\
\hline
\end{tabular}

Let $X \in\left[X_{\min }, X_{\max }\right]$. In addition to (14), we should make sure that the imaginary part of $\Gamma$ changes sign; hence from Table I, line 3 (Imag), we find:

$$
X_{\min }<X_{t}+X_{a}<X_{\max }
$$

Furthermore, one should consider $R^{\prime}$ to be adequate (not very small), so that we would get sufficient samples of $\Gamma$ in the $2^{\text {nd }}$ and $3^{\text {rd }}$ quadrants. Based on these remarks, we will select the proper components for the circuit.

\section{A. Selection of components}

Varactors typically take values up to $40 \mathrm{pF}$. As discussed earlier, the decoupling capacitance $C^{\prime}$ should be much greater than $C$. For the UHF band of $865 \mathrm{MHz}-935 \mathrm{MHz}$, feed-through capacitors should be employed, that ensure self-resonance greater than $1 \mathrm{GHz}$ and sufficiently high capacitance. Models "NFM21CC471R1H3" (470pF) and "NFM21CC221R1H3" (220pF) will be used from "Murata Manufacturing" [14]. A large selection of varactors have been acquired, including: "BB178", "BB179", "BB181" by "NXP", "1SV309", "1SV262" by "Toshiba" and "BB439", "BB640” by "Infineon" [12] - [14]. Selection of the varactor is made, depending on the antenna plus the transistor's reactance according to (15). The three available transistors were measured at the network analyzer. Their impedances change slightly from $865 \mathrm{MHz}$ to $935 \mathrm{MHz}$ and are summarized in Table II.

TABLE II. MEASURED IMPEDANCE OF TrAnSistors AT "STATE 1"

\begin{tabular}{|c|c|c|c|c|c|}
\hline Model & $\boldsymbol{X}_{\boldsymbol{t}}$ & Model & $\boldsymbol{X}_{\boldsymbol{t}}$ & Model & $\boldsymbol{X}_{\boldsymbol{t}}$ \\
\hline $\begin{array}{c}\text { Agilent } \\
\text { AT32033 }\end{array}$ & $10.1+17.5 \mathrm{i}$ & $\begin{array}{c}\text { CEL } \\
\text { NE68033 }\end{array}$ & $33.3-5.8 \mathrm{i}$ & $\begin{array}{c}\text { Zetex } \\
\text { BFS17NTA }\end{array}$ & $14+11.4 \mathrm{i}$ \\
\hline
\end{tabular}

The "A09-HSM-7" whip antenna manufactured by "Maxstream" was measured with a vector network analyzer and its impedance changes from $X_{a}{ }^{(865 M H z}=23-i 8.8(\mathrm{Ohm})$ to $X_{a}^{(930 \mathrm{MHz})}=33.8+i 17.3(\mathrm{Ohm})$ at $865 \mathrm{MHz}$ to $930 \mathrm{MHz}$ respectively. From (14) and Table I, it is found that by selecting transistor "NE68033" the real part of $\Gamma_{l}$ would never become negative; hence the vector $\Gamma_{l}$ wouldn't take values at the $2^{\text {nd }}$ and $3^{\text {rd }}$ quadrants. In order to obtain good measurements' resolution in the $2^{\text {nd }}$ and $3^{\text {rd }}$ quadrants, we have to make sure that $R^{\prime}=\left(R_{a}^{2}-R_{t}^{2}\right)^{1 / 2}$ is large enough. Hence, performing the measurements at $930 \mathrm{MHz}$ represents a better choice than $865 \mathrm{MHz}$, because $R_{a}$ is greater.

Furthermore, we find that $X_{t}+X_{a}$ at $930 \mathrm{MHz}$ for "AT32033" and "BFS17NTA" ranges from $27.7 \mathrm{Ohms}$ to $34.8 \mathrm{Ohms}$, whereas at $865 \mathrm{MHz}$ it ranges from $2.6 \mathrm{Ohms}$ to $8.7 \mathrm{Ohms}$. The impedance of available commercial varactors goes down to $X_{\min }=4 \mathrm{Ohms}(40 \mathrm{pF}$ at $930 \mathrm{MHz})$. Therefore, from (15) and the phase-changes presented in Table I, performing the measurements at $930 \mathrm{MHz}$ represents a better option, in order to ensure larger phase variations.

By evaluating all different combinations of available varactors and transistors with the above antenna, we created plots like the one shown in Figure 8. It shows the variation of the modified reflection coefficient, given in (2), by increasing the capacitance of varactor "BB179" attached to the three transistors of Table II. In this plot, each sample corresponds to a $10 \mathrm{hm}$-increment of the impedance of the employed varactor. Good measurements-resolution for the rotating $\Gamma$ vector is accomplished with the selected varactor. Notice that by employing transistor "NE68033", $\Gamma$ takes values only at the $4^{\text {th }}$ quadrant of the unit circle, as explained previously, due to the transistor's large resistance. From the comparison, it is found that "varactor" BB179" should be selected with transistor "AT32033" to offer a phase-variation of $191^{\circ}$ with magnitude ranging from 0.55 to 0.86 .

Achieved $\Gamma$ with varactor "BB179" attached to different transistors

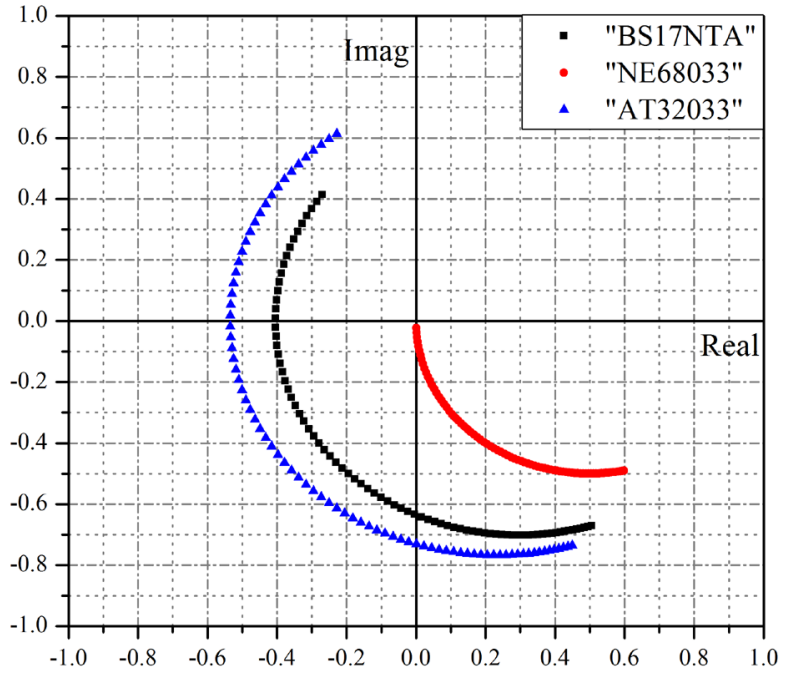

Figure 8. Variation of the modified reflection coefficient by increasing the capacitance of varactor "BB179" attached to different transistors.

\section{MEASUREMENTS}

We have constructed the proposed circuit, selecting the varactor "BB179" with the transistor "AT32033". We measured the complex impedance of the circuit for the two transistor states in the UHF RFID frequency band at the vector network analyzer. The circuit is characterized for different dcvoltages applied to the varactor, namely: $1 \mathrm{~V}, 5 \mathrm{~V}, 10 \mathrm{~V}, 15 \mathrm{~V}$, $20 \mathrm{~V}, 25 \mathrm{~V}, 28 \mathrm{~V}$. Then, the modified reflection coefficient in (2) is calculated. The results are presented in Figure 9.

Significant phase-variation is accomplished in the entire frequency band, by adjusting the DC-voltage, hence changing the tag's load. Therefore, the structural mode term of the tagantenna can be evaluated in the entire frequency band with the constructed device. Also notice that the modified reflection 
coefficient at "State 0 " of the transistor experiences small changes in the entire frequency band.

We are currently in the process of performing the backscattering measurements, in order to calculate the tagantenna's structural mode term.

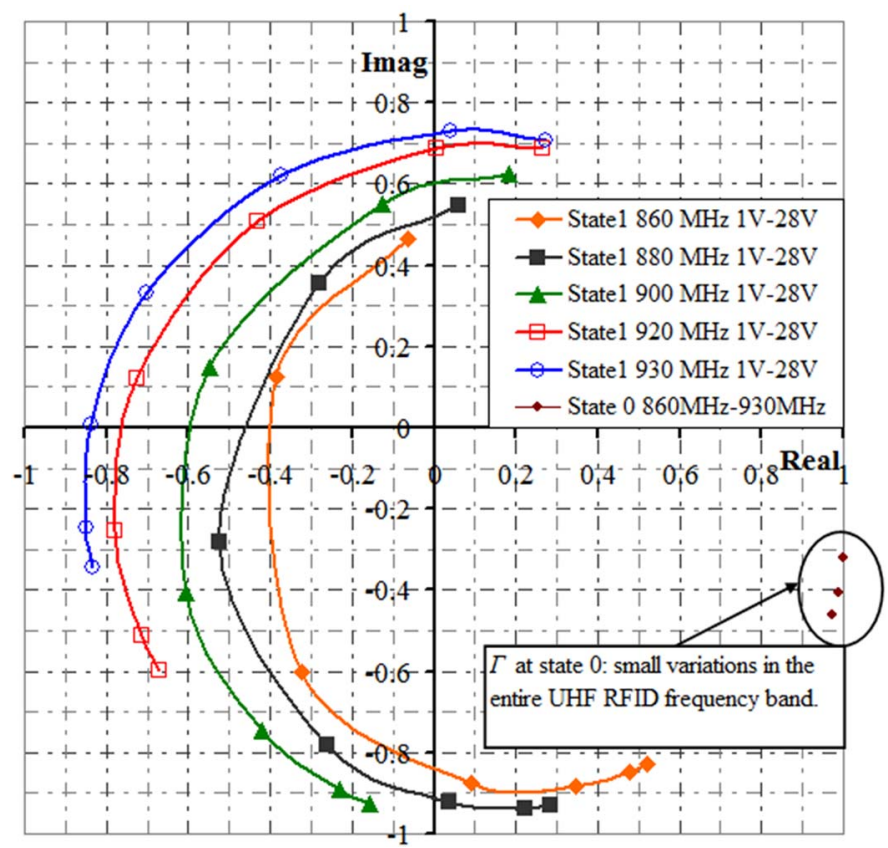

Figure 9. Measured modified reflection coefficient at the two transistor states in the UHF RFID frequency band for increasing dc-voltage at the varactor.

\section{CONCLUSIONS}

In this paper, we have presented a prototype device to measure a tag-antenna's structural-mode-term. In contrast to prior art, the proposed device can be attached to any antenna, while the measurements can be conducted under any conditions, including "rich-scattering" environments. Experimentation is expected to characterize the antenna's structural mode term vs. frequency and orientation with respect to the incident field.

Furthermore, as the proposed device provides selectable tag-loads in a continuous range, it can be used for experimentation on different modulation loading. It can also be used as a sensor, where sensing is mapped to the change of the varactor's voltage.

\section{ACKNOWLEDGEMENT}

This work was supported by projects "Thales: RFIDCORE" and "BLASE-ERC-04", executed in the context of the Education \& Lifelong Learning Program of General Secretariat for Research \& Technology (GSRT) and funded through European Union-European Social Fund and national funds.

\section{REFERENCES}

[1] A. Bletsas, A. G. Dimitriou, J. N. Sahalos, "Improving backscatter radio tag efficiency," IEEE Trans. Microwave Theory \& Techniques, vol. 58, no 6, pp. 1502-1509, June 2010.

[2] A. G. Dimitriou, A. Bletsas and J. N. Sahalos, "On the design of passive RFID tags for ASK modulation," 5th European Conference on Antennas \& Propagation (EuCAP), Rome, 11-15 April, 2011.

[3] A. G. Dimitriou, A. Bletsas and J. N. Sahalos, "Practical Considerations of ASK Modulated Passive Tags," 6th European Conference on Antennas \& Propagation (EuCAP), Prague, 26-30 March, 2012.

[4] C. A. Balanis, Antenna Theory Analysis and Design, Third Edition, New Jersey, John Wiley \& Sons, pp. 98-104, 2005.

[5] R. B. Green, The General Theory of Antenna Scattering, Dissertation, Ohio State University, 1963.

[6] R. W. P. King and C. W. Jr. Harrison, "The receiving antenna," Proc. I.R.E., no 21, January, 1944

[7] C.-C. Yen, A. E. Gutierrez, D. Veeramani, and D. van der Weide, "Radar cross-section analysis of backscattering RFID tags," IEEE Antennas Wireless Propag. Lett., vol. 6, pp. 279-281, 2007.

[8] A.H. Rida, L. Yang, S. Serkan Basat, A. Ferrer-Vidal, S. Nikolaou, M. M. Tentzeris, "Design, development and integration of novel antennas for miniaturized UHF RFID tags," IEEE Trans. Antennas Propagat., vol. 57 , no 11 , pp. $3450-3457$.

[9] G. Marrocco, "The art of UHF RFID antenna design: impedancematching and size-reduction techniques, "IEEE Antennas \& Propagat. Mag. Vol. 50, no. 1, pp. 66-79, Feb. 2008.

[10] D. M. Dobkin, The RF in RFID, Passive UHF RFID in Practice, Newnes, Elsevier, 2008.

[11] Datasheet "BB179, UHF variable capacitance diode", NXP Semiconductors.

[12] Datasheet "1SV309", UHF SHF Tuning, Toshiba.

[13] Datasheet "BB439”, Silicon Variable Capacitance Diode, Infineon.

[14] "SMD/BLOCK Type EMI Suppression Filters, EMIFIL" Catalogue, Murata Manufacturing. 